\title{
Procesos de innovación gerencial en instituciones hospitalarias de La Guajira - Colombia ${ }^{1}$
}

DOI: http://dx.doi.org/10.17981/econcuc.37.1.2016.09

\section{Mirle Irina Curiel Correa ${ }^{2}$}

\section{Resumen}

La innovación en los procesos gerenciales representa un factor determinante en las organizaciones e implica conocimiento, manejo de las necesidades y expectativas del personal, por esta razón los gerentes han emprendido esfuerzos dirigidos a fortalecerlos, lo cual debe partir de la conveniencia de tener un propósito empresarial claro, digno y compartido por todos. Por lo cual, analizar los aspectos relacionados con los tipos de innovación: innovación de procesos e innovación de naturaleza funcional permitió determinar las prácticas en innovación y modernización tecnológica en las instituciones hospitalarias de La Guajira-Colombia. Se define metodológicamente una investigación de tipo descriptivo, diseño no experimental de tipo transeccional . Los hallazgos permitieron evidenciar que el desarrollo de los procesos de I + D con aplicaciones gerenciales son escasos, sin embargo, prevalece un esfuerzo desde estas instituciones por involucrase más en los diferentes tipos de innovación, que les permita introducir cambios dirigidos a incrementar la funcionalidad y las prestaciones de la empresa para construir una base permanente de progreso.

Palabras clave: innovación gerencial; desarrollo organizacional; instituciones hospitalarias; La GuajiraColombia.

Recibido: 4.8.2015 Devuelto para revisión: 16.9.2015 Aceptado: 27.10.2015

\begin{abstract}
${ }^{1}$ Artículo científico derivado de la investigación "Innovación en Instituciones Hospitalarias Públicas Tipo II de la GuajiraColombia derivado del Proyecto de Tesis de Maestría del Programa de Gerencia De Proyectos de Investigación y Desarrollo (URBE, Venezuela). Financiada por la Universidad de la Guajira.

${ }^{2}$ Magister en Gerencia de Proyectos de Investigación y Desarrollo. Universidad Rafael Belloso Chacín, MaracaiboVenezuela. Especialista en Revisoría Fiscal. Universidad Libre, Barranquilla-Colombia. Contador Público. Universidad Autónoma del Caribe, Barranquilla-Colombia. Docente tiempo completo ocasional de la Universidad de La Guajira adscrito al grupo de investigación INNOVAR. mcuriel@uniguajira.edu.co
\end{abstract}




\title{
Processes of managerial innovation in hospitals of La Guajira - Colombia
}

\begin{abstract}
Innovation in management processes is a determining factor in organizations and implies knowledge, management of staff needs and expectations, for this reason managers have undertaken efforts aimed at strengthening them, which should start from the convenience of having a business purpose clear, dignified and shared by all. Therefore, to analyze the aspects related to the types of innovation: innovation of processes and innovation of a functional nature allowed determining the practices in innovation and technological modernization in the hospital institutions of La Guajira-Colombia. A descriptive, non-experimental, transectional type research is methodologically defined. The findings made it possible to show that the development of R \& D processes with management applications is scarce; however, an effort from these institutions prevails to be more involved in the different types of innovation, allowing them to introduce changes aimed at increasing the functionality and the benefits of the company to build a permanent basis of progress.
\end{abstract}

Keywords: managerial innovation; organizational development; hospital institutions; La GuajiraColombia.

\section{Introducción}

La innovación se ha convertido en uno de los temas de interés para todos. Esta trae consigo consecuencias ventajosas, tanto en posicionamiento global, como en incremento de capital, no sólo económico sino también de capital intelectual, el cual es hoy en día uno de los activos más importantes de cualquier organización o persona individual, como lo menciona Drucker (2000) cuando habla del knowledge worker. Sin embargo, es importante aclarar que la innovación por sí sola no garantiza el éxito; es necesario, la utilización de metodologías, estrategias, estudios de los factores que intervienen en el proceso de innovación y el estudio de los riesgos que éste puede traer. A esto se le suma la visión amplia y precisa que deben poseer los dirigentes de países o líderes de las empresas, quienes son ejes fundamentales en el proceso de innovación, puesto que son ellos los que planean, verifican y coordinan los procesos que hacen parte de 
la organización y de los externos que intervienen en ella.

La inversión en investigación y desarrollo $(\mathrm{I}+\mathrm{D})$ como porcentaje del PIB es menor, como lo es el número de patentes, y muchas empresas no cuentan con departamentos dedicados a investigación y desarrollo ( + D). Lo que debe comprenderse, sin embargo, es que estas diferencias cuantitativas reflejan diferencias más profundas. En efecto, los sistemas de innovación de los países en desarrollo son cualitativamente diferentes como resultado de desfases acumulativos con respecto a los países desarrollados. Ello no significa que estos sistemas sean irreparables sino que muestran obvias desventajas que deben reconocerse y abordarse. Por otro lado, según los indicadores del Global Competitiveness Report, el valor promedio del índice de innovación de América Latina muestra claramente un retraso con respecto a la mayor parte de las otras regiones del mundo.

En América Latina existen escasos vínculos y, por consiguiente, un débil flujo de conocimientos entre las empresas y las instituciones de investigación, incluidas las universidades. En esta encuesta también se indagó acerca del origen de sus ideas innovadoras, con lo cual 13,4\% de las empresas colombianas encuestadas se las atribuyeron a las universidades y el 7,4\% a los institutos de investigación del sector público.

Sin embargo, para el 45\% de las empresas de más de 50 o menos de 100 empleados, que además se incluyen dentro de la categoría de empresas innovadoras de calibre internacional, la fuente de ideas innovadoras son las universidades, y para el $43 \%$ lo son las instituciones públicas de investigación.

En el marco del sector salud, Colombia busca innovar el servicio de salud que consiste en un conjunto de instituciones y organismos cuya finalidad primordial era procurar la salud de la comunidad, articulados bajo un modelo centralista en las decisiones políticas y en la planeación y asignación de recursos, pero con un esquema de delegación de la nación en los departamentos con respecto a la organización y prestación de servicios. En tal sentido, en Colombia, al inicio de la década de los 90 se promulgó la Ley Nacional de Ciencia y Tecnología (Ley 29 de 1990), mediante la cual se consolidó el Sistema Nacional de Ciencia y Tecnología, incorporando adicionalmente el Sistema Nacional de Innovación, con el propósito de incrementar la productividad y la competitividad de las empresas y del sector productivo nacional en su conjunto, lo que representa un elemento clave dentro de las nuevas estrategias (Colciencias, 2005). 
Las organizaciones hospitalarias se consideran un elemento fundamental de desarrollo, a su vez también, un indicador del nivel de progreso de un país. Los grandes retos que se deben asumir son la inequidad social y la corrupción de los dirigentes políticos y administrativos, actores del sistema del sector salud, lo que conlleva a las grandes brechas de información, de construcción, de generación y de aplicación de conocimientos.

En Colombia, la reforma de la seguridad social generó un cambio significativo en la prestación de los servicios de salud con el objetivo de que las instituciones públicas hospitalarias se desarrollen como organizaciones de alta competitividad, con estructuras flexibles y dinámicas con capacidad tecnológica estructural y de dirección para ofrecer servicios con calidad y eficiencia, teniendo en cuenta la satisfacción del usuario y el mejoramiento de la productividad, para lo cual debieron convertirse en Empresas Sociales del Estado, convirtiendo los viejos recursos de la oferta a la demanda y obtener sus ingresos por la venta de servicios a las diversas administradoras del régimen subsidiado y contributivo, pilares de la Ley 100 de 1993.
En el departamento de La Guajira (Colombia), según la agenda prospectiva (2002), se pone en evidencia la carencia de una visión de futuro que oriente el desarrollo sectorial de los procesos de innovación en las instituciones hospitalarias con base en las características del entorno y fije de forma prioritaria y concertada una política científica y de innovación regional, todo con el objeto fundamental de rentabilizar los resultados de I + D que generen los centros de investigación y, además, establezca una política tecnológica que aumente el valor agregado del sector salud de la región.

Desde esta perspectiva, los procesos de innovación gerencial en las instituciones hospitalarias del sector salud constituyen un eje estratégico pertinente y relevante de cambio creativo, que implica reinventar y renovar las estructuras, los procesos, los sistemas y maneras de medir los resultados y el desempeño de las organizaciones. En tal sentido, la innovación está enfocada a los resultados, que se miden de acuerdo con la satisfacción del usuario. En consecuencia surge el siguiente planteamiento problémico central: ¿cuál es la naturaleza funcional y el nivel de desarrollo de los procesos de innovación gerencial en las instituciones hospitalarias públicas tipo II de La Guajira colombiana? 
Referentes teóricos y empíricos de los procesos de innovación gerencial

Las principales tendencias identificadas en la literatura científica permiten poner en evidencia que el concepto de la innovación gerencial comprende un proceso o conjunto de acciones tácticas y estratégicas a partir de las cuales la generación del nuevo conocimiento científico y tecnológico promueve una apropiación novedosa que tiene impacto en la gestión y desarrollo organizacional bajo un marco riguroso de aseguramiento de la calidad y la necesidad de desarrollar nuevas prácticas cada vez más eficientes y eficaces (Gaynor, 1999; Pavón y Goodman, 1981).

Esta conceptualización central supone un proceso de cambio que se podría considerar microeconómico; el cambio tiene también un importante componente macroeconómico, ya que es objetivo principal convertir esas mejoras empresariales o cambios globales para la sociedad, y para ello se debe difundir la innovación. De acuerdo con su naturaleza las innovaciones podrían clasificarse en: tecnológicas, comerciales, administrativas, financieras y de recursos humanos (Booz, Allen y Hamilton, 1982; Drucker, 2000).

En complemento se resalta el aporte de Martínez (2000), quien plantea que la in- novación gerencial es una actividad que consiste en la introducción de nuevas combinaciones de factores que aseguren un mayor nivel de eficacia de un servicio, por otra parte, esto incrementa la eficacia en la utilización de los factores productivos de bienes 0 servicios, conseguida mediante la aplicación de nuevas técnicas y adelantos tecnológicos, que, atendiendo a su originalidad, pueden ser innovaciones incrementales o radicales.

La mayoría de los procesos de innovación se incorporan dentro de los lineamientos del mejoramiento continuo. Desde esta perspectiva surge el concepto de innovación incremental, comprendido como el conjunto de mejoras o transformaciones derivadas de la tecnología existente, introduciendo cambios en los productos o procesos actuales y generando a su vez un valor agregado al potencial del diseño organizacional establecido. Es así como Mansfield (1988) argumenta que durante la introducción de un nuevo producto al mercado, las mejoras en el proceso, las funcionalidades, la estrategia o en el producto pueden ser casi tan importantes como la nueva idea en sí. En consecuencia, la importancia de las innovaciones incrementales de ningún modo minimiza el efecto de las innovaciones radicales.

En tal sentido, un proceso gerencial podría considerarse de innovación radical si el conocimiento tecnológico necesario para 
explotarlo es muy diferente del conocimiento existente y lo hace obsoleto, generando una nueva práctica y requiriendo a su vez un marco de competencias organizacionales diferentes. En tal sentido, la innovación radical es algo nuevo para el mundo, constituye una ruptura profunda con las formas establecidas de hacer las cosas, suele abrir nuevos mercados y aplicaciones potenciales, cambia las bases de la competencia, crea grandes dificultades a las empresas establecidas y puede suponer la base para la entrada con éxito de nuevas empresas e incluso la redefinición de la industria. En algunos casos las innovaciones radicales surgen derivadas de las actividades de transferencia de tecnología que aprovechan las oportunidades de introducir una tecnología ya disponible y válida (normalmente con alguna modificación que la adapte a las condiciones locales) en áreas geográficas cuya aptitud para la misma no había sido anteriormente reconocida ni utilizada (Tushman y Anderson, 1986; Baumol, 1993).

Un factor fundamental del presente artículo comprende la conceptualización de la innovación gerencial vista como proceso $\mathrm{y}$, por lo tanto, cabe resaltar los aportes de Fernández (2005) quien considera la innovación como un proceso constante, susceptible de planificación, programación y control que puede desagregarse en actividades independientes para simplificar su gestión. Este proceso, se sustenta en los avances científicos y tecnológicos inicialmente derivados de la investigación básica y los desarrollos de prototipos experimentales, los cuales, a su vez, sirven como base para la implementación de procesos de investigación aplicada, desarrollo, producción y comercialización. En esta línea de desarrollo organizacional, Vaston (1988) considera que los procesos de innovación representan un área de gran relevancia en la mejora de la velocidad del cambio organizacional y su impacto esperado sobre el medio, rompiendo paradigmas y modificando principalmente de forma estructural y funcional aquellos procesos que son demasiados rígidos y burocráticos.

La innovación incide adicionalmente en los procesos de reposicionamiento de los productos y servicios. Por ejemplo, los fabricantes de hornos microondas habían concebido el producto para sustituir la cocina eléctrica o de gas, pero esta idea no tuvo éxito en el mercado. Posteriormente, rediseñaron el producto para reducir su tamaño y ofrecer así un accesorio novedoso y complementario de las cocinas tradicionales (Robertson, 1993).

En muchos casos, las ideas de los clientes se canalizan a través de los vendedores, quienes, a su vez, también constituyen otra 
fuente de ideas para la innovación. Por ejemplo, las iniciativas comerciales llevadas a cabo por Marconi, cuyos primeros clientes fueron el ejército y la armada inglesa, descubrieron nuevos mercados para la radio y plantearon desafíos tecnológicos que tuvieron que ser investigados con el objeto de solucionar los problemas encontrados y para los que aún no existían conocimientos científicos (Basalla, 1988).

Las características de algún tipo de innovaciones, como por ejemplo, las de carácter radical, aconsejan su internalización para minimizar los costes de transacción. La innovación radical es muy incierta, lo que hace muy difícil la especificación de los estados futuros del mundo y deja un gran espacio para el oportunismo. A causa de su naturaleza tácita o del volumen total de parte de la información, puede ser difícil especificar en contratos qué es lo que se espera de cada parte, ya que hay asimetría de información. Con frecuencia, este tipo de innovación requiere fábricas o individuos especializados, lo que de nuevo aumenta la probabilidades del oportunismo (Afuah, 1998).

Una perspectiva opuesta corresponde la externalización que, de acuerdo con Robertson (1993), se trata de procesos innovadores de tecnologías específicas para la empresa que se combinan entre ellas para generar unos resultados únicos y más valiosos que el conjunto de resultados que se obtendrían de cada una de ellas si se utilizaran por separado. Por otra parte, se trata de tecnologías difíciles de imitar por los competidores. Finalmente, estas tecnologías las tiene que desarrollar internamente la organización, ya que no se pueden comprar y vender en el mercado. Estas tecnologías trabajando juntas constituyen el potencial tecnológico de la empresa y la fuente de su ventaja competitiva.

En el presente artículo, se han identificado cinco clases de roles que desempeñan los individuos clave en el reconocimiento del potencial de una innovación y su explotación: generador de ideas, portero (o extensor de fronteras), emprendedor (o campeón de producto), patrocinador y gerente de proyecto (Roberts y Fusfeld, 1981). Cuantos más efectivos sean estos individuos, más innovadora será la empresa. Ahora bien, cuando alguno de ellos abandona la organización suele ser difícil encontrar un sustituto, ya que trabajadores nuevos no pueden desempeñar estos mismos roles. En síntesis, el concepto de innovación de Fernández (2005) va más allá de los puros cambios técnicos para comprender también la promoción, la distribución y muchas formas en las que las empresas pueden conceder ventajas competitivas sobre sus rivales. 
A continuación se plantean las principales especificaciones metodológicas consideradas para abordar el planteamiento del problema objeto de estudio.

\section{Método}

La metodología comprendió un diseño no experimental de tipo transeccional, con nivel de alcance de corte descriptivo. Esta propuesta metodológica responde de forma sistemática y coherente al problema formulado (Ballestrini, 2001; Méndez, 2001; Hernández, Fernández y Baptista, 2006).

Muestra. Los 32 participantes del presente estudio conformaron una muestra tipo de los actores centrales de los procesos de innovación gerencial en las diferentes instituciones hospitalarias tipo II en Riohacha y Maicao (La Guajira).

Tabla 1

Marco muestral.

\begin{tabular}{lccc}
\hline $\begin{array}{l}\text { Instituciones } \\
\text { hospitalarias }\end{array}$ & Directores & $\begin{array}{c}\text { Jefes de } \\
\text { área }\end{array}$ & Total \\
\hline Riohacha & 1 & 14 & 15 \\
Maicao & 1 & 16 & 17 \\
Total & 2 & 30 & 32 \\
\hline
\end{tabular}

Fuente: elaboración propia
Instrumento. Se diseñó una encuesta con el formato de escala tipo Likert con cinco (5) grados, desde el total desacuerdo hasta el total acuerdo, orientada a la caracterización de los procesos de innovación gerencial en las instituciones hospitalarias. La confiabilidad obtenida fue alta (a:.94), tomando como referencia que a partir de (a:.80) se considera que los instrumentos de este tipo reportan evidencia de su consistencia interna.

Procedimiento. Inicialmente se diseñó el instrumento, con su posterior valoración por jueces expertos y prueba piloto. En segunda instancia, se administró a los 32 participantes en representación de las instituciones hospitalarias tipo II en Riohacha y Maicao (La Guajira), asumiendo el protocolo de las consideraciones éticas en lo referente con el consentimiento informado previo y el aseguramiento de la confidencialidad de los participantes y las instituciones.

\section{Resultados}

En el presente apartado, se ilustran los principales hallazgos relacionados con el análisis de los procesos de innovación gerencial en las instituciones hospitalarias públicas tipo II de La Guajira colombiana. 
Tabla 2

Tipos de innovación en los procesos gerenciales.

\begin{tabular}{|c|c|c|c|c|c|c|c|c|c|c|}
\hline \multirow{2}{*}{ Ítems } & \multicolumn{2}{|c|}{ TA(1) } & \multicolumn{2}{|c|}{ MA(2) } & \multicolumn{2}{|c|}{ NAND(3) } & \multicolumn{2}{|c|}{ MD(4) } & \multicolumn{2}{|c|}{ TD(5) } \\
\hline & FA & $\%$ & FA & $\%$ & FA & $\%$ & FA & $\%$ & $\overline{\text { FA }}$ & $\%$ \\
\hline \multicolumn{11}{|l|}{ Indicador: incremental } \\
\hline $\begin{array}{l}\text { 1. En el hospital se desarrollan actividades de I + D para } \\
\text { obtener mejoras en la tecnología ya existente. }\end{array}$ & 4 & 12,5 & 9 & 28,1 & 8 & 25,0 & 6 & 18,8 & 5 & 15,6 \\
\hline $\begin{array}{l}\text { 2. En las instituciones hospitalarias se realizan } \\
\text { periódicamente mejoras en los procesos actuales. }\end{array}$ & 2 & 6,3 & 6 & 18,8 & 9 & 28,1 & 7 & 21,9 & 8 & 25,0 \\
\hline $\begin{array}{l}\text { 3. En las instituciones hospitalarias los cambios han } \\
\text { permitido mejorar la calidad del servicio de salud prestado. }\end{array}$ & 4 & 12,5 & 7 & 21,9 & 8 & 25,0 & 5 & 15,6 & 8 & 25,0 \\
\hline \multicolumn{11}{|l|}{ Indicador: radical } \\
\hline $\begin{array}{l}\text { 4. En los hospitales se han desarrollado cambios radicales } \\
\text { a tecnologías existentes. }\end{array}$ & 5 & 15,6 & 6 & 18,8 & 9 & 28,1 & 8 & 25,0 & 4 & 12,5 \\
\hline $\begin{array}{l}\text { 5. A las instituciones hospitalarias les ha resultado difícil } \\
\text { romper con las rutinas y procedimientos de las viejas } \\
\text { tecnologías. }\end{array}$ & 4 & 12,5 & 7 & 21,9 & 7 & 21,9 & 10 & 31,3 & 4 & 12,5 \\
\hline $\begin{array}{l}\text { 6. Las instituciones hospitalarias invierten en innovaciones } \\
\text { radicales en toda la organización. }\end{array}$ & 5 & 15,6 & 6 & 18,8 & 9 & 28,1 & 8 & 25,0 & 4 & 12,5 \\
\hline \multicolumn{11}{|l|}{ Indicador: original } \\
\hline $\begin{array}{l}\text { 7. Las innovaciones desarrolladas en las instituciones } \\
\text { hospitalarias han permitido el incremento de la eficacia } \\
\text { del personal. }\end{array}$ & 4 & 12,5 & 7 & 21,9 & 8 & 25,0 & 7 & 21,9 & 6 & 18,8 \\
\hline $\begin{array}{l}\text { 8. Las instituciones hospitalarias destinan recursos } \\
\text { suficientes para desarrollar innovaciones internamente. }\end{array}$ & 2 & 6,3 & 9 & 28,1 & 8 & 25,0 & 4 & 12,5 & 9 & 28,1 \\
\hline $\begin{array}{l}\text { 9. En las instituciones hospitalarias existen programas de } \\
\text { capacitación para las innovaciones originales. }\end{array}$ & 4 & 12,5 & 5 & 15,6 & 14 & 43,7 & 7 & 21,9 & 2 & 6,3 \\
\hline \multicolumn{11}{|l|}{ Indicador: adaptada } \\
\hline $\begin{array}{l}\text { 10. Las instituciones hospitalarias adaptan tecnologías } \\
\text { para hacer eficaces los procesos. }\end{array}$ & 5 & 15,6 & 6 & 18,8 & 9 & 28,1 & 8 & 25,0 & 4 & 12,5 \\
\hline $\begin{array}{l}\text { 11. La mayoría de las innovaciones en las instituciones } \\
\text { hospitalarias son adaptadas. }\end{array}$ & 1 & 3,1 & 9 & 28,1 & 9 & 28,1 & 5 & 15,6 & 8 & 25,1 \\
\hline $\begin{array}{l}\text { 12. En las instituciones hospitalarias se hacen estudios de } \\
\text { factibilidad para verificar la eficacia de las innovaciones } \\
\text { adaptadas. }\end{array}$ & 9 & 28,1 & 5 & 15,6 & 5 & 15,6 & 7 & 21,9 & 6 & 18,8 \\
\hline
\end{tabular}

Fuente: elaboración propia. 
De los resultados presentes en la tabla 2 , con relación a la dimensión tipos de innovación que representa al indicador innovación incremental, se enfatiza que el 12,5\% de los funcionarios encuestados está totalmente de acuerdo con que en el hospital se desarrollan actividades de I + D para obtener mejoras en la tecnología ya existente y el $28,1 \%$ está medianamente de acuerdo. Frente a la afirmación según la cual, en las instituciones hospitalarias se realizan periódicamente mejoras en los procesos actuales, el $21,9 \%$ se encuentra medianamente en desacuerdo y un $25,0 \%$ estuvo totalmente en desacuerdo.

Asímismo, sobre el hecho de que en las instituciones hospitalarias los cambios han permitido mejorar la calidad del servicio de salud prestados, los resultados demuestran que el $12,5 \%$ estuvo totalmente de acuerdo, el $21,9 \%$ medianamente de acuerdo, el $25,0 \%$ ni de acuerdo ni en desacuerdo, el $15,6 \%$ medianamente en desacuerdo y el $25,0 \%$ totalmente en desacuerdo.

Con relación al indicador innovación radical, se pudo observar que solamente el $15,6 \%$ de los funcionarios encuestados manifestó estar totalmente de acuerdo con que en los hospitales se han desarrollado cambios radicales a tecnologías existentes. Respecto a si las instituciones hospitalarias invierten en innovaciones radicales en toda la organización, el 25,0\% se encuentra medianamente en desacuerdo y el 12,5\% está totalmente en desacuerdo.

Según el indicador original, en cuanto a si las innovaciones desarrolladas en las instituciones hospitalarias han permitido el incremento de la eficacia del personal, se observó que solamente el $12,5 \%$ de los sujetos encuestados manifestó estar totalmente de acuerdo y el $21,9 \%$ medianamente de acuerdo, principalmente. Igualmente, se pudo evidenciar que solamente un $6,3 \%$ de los sujetos encuestados está totalmente de acuerdo con que las instituciones hospitalarias destinan recursos suficientes para desarrollar internamente innovaciones $\mathrm{y}$ el 28,1\% reportó estar medianamente de acuerdo. Para el ítem de si en las instituciones hospitalarias existen programas de capacitación para las innovaciones originales, se resalta que el $43,7 \%$ se encuentra neutral, mientras que un $21,9 \%$ está medianamente en desacuerdo y el $28,1 \%$ está totalmente en desacuerdo, principalmente.

Para el indicador innovación adaptada, se puso en evidencia que el $15,6 \%$ está totalmente de acuerdo con que las instituciones hospitalarias adaptan tecnologías para hacer eficaces los procesos y el 18,8\% se encuentra medianamente de acuerdo. Con relación a la información según la cual la mayoría de las innovaciones en las institu- 
ciones hospitalarias son adaptadas, solamente un $3,1 \%$ está totalmente de acuerdo y el $28,1 \%$ esta medianamente de acuerdo. Referente al ítem 12, el 28,1\% manifestó estar totalmente de acuerdo en que en las instituciones hospitalarias se hacen estudios de factibilidad para verificar la eficacia de las innovaciones adaptadas y el 15,6\% manifestó estar medianamente de acuerdo, en contraste con, el $21,9 \%$ medianamente en desacuerdo y el 18,8\% totalmente en desacuerdo.

\section{Dimensión: procesos.}

- Indicadores: cadena, eslabón, internalizar, externalizar, roles personales.

Al observar la tabla 3, que refiere a la dimensión procesos, en el indicador cadena, se pudo establecer principalmente que el $12,5 \%$ de los funcionarios encuestados está totalmente de acuerdo con que se determinan los cambios en los procesos y se evalúan los beneficios para las instituciones hospitalarias, unido al 18,8\% que está medianamente de acuerdo. Además, se pudo poner en evidencia que el $15,6 \%$ de los encuestados está totalmente de acuerdo en que se genera valor agregado al producto de servicio en las instituciones hospitalarias y un $25,1 \%$ estuvo medianamente de acuerdo.
De igual manera, en lo que se refiere al indicador eslabón, se observó que sólo un $9,4 \%$ de los funcionarios encuestados está totalmente de acuerdo con que se realizan chequeos a las herramientas de gestión y procedimientos administrativos y de medio ambiente desarrolladas e implementadas en los hospitales. En complemento, el 25,0\% de los encuestados está totalmente de acuerdo con la afirmación según la cual se prioriza un plan de acción correspondiente a las instituciones hospitalarias. Respecto a si se genera conocimiento de la detección de los principales aspectos medioambientales que afectan a las instituciones hospitalarias, el $12,5 \%$ de los sujetos encuestados respondió estar totalmente de acuerdo y el $28,1 \%$ medianamente de acuerdo, en contraste con el $25,0 \%$ medianamente en desacuerdo y el $6,3 \%$ totalmente en desacuerdo.

Referente al indicador internalizar, al evaluar si están integrados en las instituciones hospitalarias los procesos, el 12,5\% de los sujetos encuestados manifestó estar totalmente de acuerdo y el 28,1\% medianamente de acuerdo. De la misma manera, se observó que el 15,6\% de los encuestados, señaló estar totalmente de acuerdo en que en las instituciones hospitalarias existe personal clave para realizar funciones que los proveedores externos no logran satisfacer. Frente a la opinión sobre si en las instituciones hospitalarias se utilizan servicios 
Tabla 3

Análisis de la innovación gerencial vista como proceso.

\begin{tabular}{|c|c|c|c|c|c|c|c|c|c|c|}
\hline \multirow{2}{*}{ Ítems } & \multicolumn{2}{|c|}{ TA(1) } & \multicolumn{2}{|c|}{ MA(2) } & \multicolumn{2}{|c|}{ NAND(3) } & \multicolumn{2}{|c|}{ MD(4) } & \multicolumn{2}{|c|}{ TD(5) } \\
\hline & FA & $\%$ & $\mathrm{FA}$ & $\%$ & $\mathrm{FA}$ & $\%$ & FA & $\%$ & $\mathrm{FA}$ & $\%$ \\
\hline \multicolumn{11}{|l|}{ Indicador: cadena } \\
\hline $\begin{array}{l}\text { 13. Se determinan los cambios en los procesos y se evalúan los } \\
\text { beneficios para las instituciones hospitalarias. }\end{array}$ & 4 & 12,5 & 6 & 18,8 & 13 & 40,6 & 8 & 25,0 & 1 & 3,1 \\
\hline $\begin{array}{l}\text { 14. Se genera valor agregado al producto de servicio en las } \\
\text { instituciones hospitalarias. }\end{array}$ & 5 & 15,6 & 8 & 25,1 & 5 & 15,6 & 5 & 15,6 & 9 & 28,1 \\
\hline \multicolumn{11}{|l|}{ Indicador: eslabón } \\
\hline $\begin{array}{l}\text { 15. Se realizan chequeos a las herramientas de gestión y } \\
\text { procedimientos administrativos y de medio ambiente desarrolladas e } \\
\text { implementadas en los hospitales }\end{array}$ & 3 & 9,4 & 5 & 15,6 & 13 & 40,6 & 6 & 18,8 & 5 & 15,6 \\
\hline $\begin{array}{l}\text { 16. Se prioriza un plan de acción correspondiente a las instituciones } \\
\text { hospitalarias. }\end{array}$ & 8 & 25,0 & 7 & 21,9 & 7 & 21,9 & 5 & 15,6 & 5 & 15,6 \\
\hline $\begin{array}{l}\text { 17. Se genera conocimiento de la detección de los principales aspectos } \\
\text { medioambientales que afectan a las instituciones hospitalarias. }\end{array}$ & 4 & 12,5 & 9 & 28,1 & 9 & 28,1 & 8 & 25,0 & 2 & 6,3 \\
\hline \multicolumn{11}{|l|}{ Indicador: internalizar } \\
\hline 18. Están integrados en las instituciones hospitalarias los procesos. & 4 & 12,5 & 9 & 28,1 & 9 & 28,1 & 8 & 25,0 & 2 & 6,3 \\
\hline $\begin{array}{l}\text { 19. En las instituciones hospitalarias existe personal clave para } \\
\text { realizar funciones que los proveedores externos no logran satisfacer. }\end{array}$ & 5 & 15,6 & 6 & 18,0 & 8 & 25,0 & 6 & 18,8 & 7 & 21,9 \\
\hline $\begin{array}{l}\text { 20. En las instituciones hospitalarias se utilizan servicios } \\
\text { temporales o de insourcing para desarrollar los procesos. }\end{array}$ & 2 & 6,3 & 7 & 21,9 & 9 & 28,1 & 8 & 25,0 & 6 & 18,8 \\
\hline \multicolumn{11}{|l|}{ Indicador: externalizar } \\
\hline $\begin{array}{l}\text { 21. En las instituciones hospitalarias para la realización de tareas } \\
\text { de apoyo frecuentemente contratan con empresas prestadoras de } \\
\text { servicio. }\end{array}$ & 4 & 12,5 & 10 & 31,3 & 9 & 28,1 & 7 & 21,9 & 2 & 6,3 \\
\hline $\begin{array}{l}\text { 22. Se evalúa permanentemente la calidad de los servicios prestados } \\
\text { por la empresa externa. }\end{array}$ & 2 & 6,3 & 8 & 25,0 & 10 & 31,3 & 9 & 28,1 & 3 & 9,4 \\
\hline \multicolumn{11}{|l|}{ Indicador: roles personales } \\
\hline $\begin{array}{l}\text { 23. Los funcionarios de los hospitales tienen un nivel de } \\
\text { especialización acorde a la tecnología utilizada. }\end{array}$ & 3 & 9,4 & 9 & 28,1 & 12 & 37,5 & 4 & 12,5 & 4 & 12,5 \\
\hline $\begin{array}{l}\text { 24. Las instituciones hospitalarias cuentan con programas de } \\
\text { capacitación para el personal. }\end{array}$ & 6 & 18,8 & 7 & 21,9 & 9 & 28,1 & 4 & 12,5 & 6 & 18,8 \\
\hline $\begin{array}{l}\text { 25. Las instituciones hospitalarias ofrecen al personal oportunidades } \\
\text { para desarrollar sus habilidades. }\end{array}$ & 3 & 9,4 & 9 & 28,1 & 6 & 18,8 & 10 & 31,3 & 4 & 12,5 \\
\hline $\begin{array}{l}\text { 26. Las instituciones tienen el personal suficientemente calificado } \\
\text { para desarrollar actividades de I + D. }\end{array}$ & 6 & 18,8 & 4 & 12,5 & 10 & 31,3 & 8 & 25,0 & 4 & 12,5 \\
\hline
\end{tabular}

Fuente: elaboración propia 
temporales o de insourcing para desarrollar los procesos, el 6,3\% de los funcionarios encuestados manifestó estar totalmente de acuerdo y el $21,9 \%$ estuvo medianamente de acuerdo.

Con referencia al indicador externalizar, se observó que el $12,5 \%$ está totalmente de acuerdo con el hecho de que en las instituciones hospitalarias se contrate la realización de tareas de apoyo con empresas prestadoras de servicios, unido al $31,3 \%$ que estuvo medianamente de acuerdo. Respecto con el hecho de que si se evalúa permanentemente la calidad de los servicios prestados por la empresa externa, sólo el 6,3\% de los funcionarios está totalmente de acuerdo, el 25,0\% medianamente de acuerdo, el $31,3 \%$ ni de acuerdo ni en desacuerdo, el $28,1 \%$ medianamente en desacuerdo y el 9,4\% totalmente en desacuerdo.

En el mismo orden, con respecto al indicador roles personales, solamente un $9,4 \%$ de los encuestados señaló estar totalmente de acuerdo con el hecho de que los funcionarios de las instituciones hospitalarias tienen un nivel de especialización acorde a la tecnología utilizada. Referente a si se percibe que en las instituciones hospitalarias cuentan con programas de capacitación para el personal, el 18,8\% está totalmente de acuerdo, unido al 21,9\% que está medianamente de acuerdo.

\section{Dimensión: naturaleza funcional}

- Indicadores: tecnológicas, comerciales, administrativas, financieras, recursos humanos.

Al analizar los datos de la tabla 4, correspondientes a la dimensión naturaleza funcional, con respecto al indicador tecnológicas, se puso en evidencia principalmente que el 3,1\% de los funcionarios está totalmente de acuerdo con que los servicios muestran el grado de novedades establecidas por las instituciones hospitalarias y el $31,3 \%$ se encuentra medianamente de acuerdo. Referente a si las instituciones hospitalarias tienen una tecnología bien tecnificada para la prestación de sus servicios, sólo el 9,4\% de los funcionarios encuestados manifestó estar totalmente de acuerdo y el $25,0 \%$ reportó estar medianamente de acuerdo. Con relación a que si la alta administración gestiona la sustitución de la tecnología presente por tecnología de punta para adaptarla al servicio que presta, el 15,6\% reportó estar medianamente en desacuerdo y el $25,0 \%$ totalmente en desacuerdo.

$\mathrm{Al}$ evaluar el indicador comerciales, en el ítem con respecto a si se implementan estrategias en la prestación de servicios en los hospitales, solamente un $6,3 \%$ está totalmente de acuerdo y el 37,5\% medianamente de acuerdo. Además, se observó que 
Tabla 4

Naturaleza funcional de los procesos de innovación gerencial

\begin{tabular}{|c|c|c|c|c|c|c|c|c|c|c|}
\hline \multirow{2}{*}{ Ítems } & \multicolumn{2}{|c|}{ TA(1) } & \multicolumn{2}{|c|}{ MA(2) } & \multicolumn{2}{|c|}{ NAND(3) } & \multicolumn{2}{|c|}{ MD(4) } & \multicolumn{2}{|c|}{ TD(5) } \\
\hline & FA & $\%$ & FA & $\%$ & FA & $\%$ & FA & $\%$ & FA & $\%$ \\
\hline \multicolumn{11}{|l|}{ Indicador: tecnológicas } \\
\hline $\begin{array}{l}\text { 27. Los servicios muestran el grado de novedades esta- } \\
\text { blecidas por las instituciones hospitalarias. }\end{array}$ & 1 & 3,1 & 10 & 31,3 & 12 & 37,5 & 5 & 15,6 & 4 & 12,5 \\
\hline $\begin{array}{l}\text { 28. Tienen las instituciones hospitalarias una tecnolo- } \\
\text { gía bien tecnificada para la prestación de sus servicios. }\end{array}$ & 3 & 9,4 & 8 & 25,0 & 11 & 34,4 & 6 & 18,8 & 4 & 12,5 \\
\hline $\begin{array}{l}\text { 29. La alta administración gestiona la sustitución de la } \\
\text { tecnología presente por tecnología de punta para adap- } \\
\text { tarla al servicio que presta. }\end{array}$ & 5 & 15,6 & 5 & 15,6 & 9 & 28,1 & 5 & 15,6 & 8 & 25,0 \\
\hline \multicolumn{11}{|l|}{ Indicador: comerciales } \\
\hline $\begin{array}{l}\text { 30. Se implementan estrategias en la prestación de ser- } \\
\text { vicios en los hospitales. }\end{array}$ & 2 & 6,3 & 12 & 37,5 & 7 & 21,9 & 7 & 21,9 & 4 & 12,5 \\
\hline $\begin{array}{l}\text { 31. Tienen las instituciones hospitalarias estrategias } \\
\text { definidas para la venta de los servicios que presta. }\end{array}$ & 3 & 9,4 & 2 & 6,3 & 12 & 37,5 & 9 & 28,1 & 6 & 18,8 \\
\hline \multicolumn{11}{|l|}{ Indicador: administrativas } \\
\hline $\begin{array}{l}\text { 32. Dentro de las instituciones hospitalarias de recha- } \\
\text { zan las conductas poco éticas. }\end{array}$ & 6 & 18,8 & 6 & 18,8 & 8 & 25,0 & 6 & 18,8 & 6 & 18,8 \\
\hline $\begin{array}{l}\text { 33. Dentro de los objetivos de las instituciones son con- } \\
\text { siderados normas, mecanismos, procedimientos y el in- } \\
\text { tercambio entre las personas que laboran. }\end{array}$ & 5 & 15,6 & 10 & 31,3 & 7 & 21,9 & 5 & 15,6 & 5 & 15,6 \\
\hline \multicolumn{11}{|l|}{ Indicador: financieras } \\
\hline $\begin{array}{l}\text { 34. Existen barreras en las instituciones hospitalarias } \\
\text { para aspectos relacionados con la venta de servicios } \\
\text { considerando la actividad económica de la entidad. }\end{array}$ & 2 & 6,3 & 6 & 18,8 & 10 & 31,3 & 9 & 28,1 & 5 & 15,6 \\
\hline $\begin{array}{l}\text { 35. Tiene la institución innovaciones que le permiten } \\
\text { mejorar los servicios para obtener mayores ingresos. }\end{array}$ & 4 & 12,5 & 8 & 25,0 & 11 & 34,4 & 4 & 12,5 & 5 & 15,6 \\
\hline \multicolumn{11}{|l|}{ Indicador: recursos humanos } \\
\hline $\begin{array}{l}\text { 36. Se incentiva a los empleados de las instituciones } \\
\text { hospitalarias para ser más pensadores y más creativos. }\end{array}$ & 5 & 15,6 & 6 & 18,8 & 9 & 28,1 & 9 & 28,1 & 3 & 9,4 \\
\hline $\begin{array}{l}\text { 37. Todo el personal de las instituciones hospitalarias } \\
\text { tienen las mismas oportunidades para introducir me- } \\
\text { joras o cambios. }\end{array}$ & 4 & 12,5 & 7 & 21,9 & 10 & 31,3 & 5 & 15,6 & 6 & 18,8 \\
\hline
\end{tabular}

Fuente: elaboración propia. 
solamente el 9.4\% de los funcionarios señaló estar totalmente de acuerdo con que las instituciones hospitalarias tienen estrategias definidas para la venta de los servicios que presta, unido a un $6,3 \%$ que se encuentra medianamente de acuerdo.

Respecto al indicador administrativas, se observó que el 18,8\% de los funcionarios encuestados está totalmente de acuerdo con que dentro de las instituciones hospitalarias se rechazan las conductas poco éticas, unido al $18,8 \%$ que se encontró medianamente de acuerdo. Por otro lado, el 15,6\% de los funcionarios está totalmente de acuerdo con que dentro de los objetivos de las instituciones son consideradas las normas, mecanismos, procedimientos y el intercambio entre las personas que laboran, y un 31,3\% manifestó estar medianamente de acuerdo.

Con respecto a la evaluación del indicador financieras, con respecto a si existen barreras en las instituciones hospitalarias para aspectos relacionados con la venta de productos considerando la actividad económica de la entidad, el $28,1 \%$ reportó estar medianamente en desacuerdo y el 15,6\% totalmente en desacuerdo. Además, se observó que el $12,5 \%$ de los funcionarios está totalmente de acuerdo con que la institución tiene innovaciones que le permiten mejorar los servicios para obtener mayores ingresos, y un $25,0 \%$ reportó estar medianamente de acuerdo.
En el mismo orden, respecto al indicador recursos humanos, $15,6 \%$ de los encuestados señaló estar totalmente de acuerdo con que se incentiva a los empleados de las instituciones hospitalarias para ser más pensadores y más creativos; unido al 18,8\% que reportó estar medianamente de acuerdo. En lo referente a si el personal de las instituciones hospitalarias tiene las mismas oportunidades para introducir mejoras o cambios, el 12,5\% está totalmente de acuerdo y el $21,9 \%$ reportó estar medianamente de acuerdo.

\section{Discusión}

En la economía mundial de hoy, en la que la innovación impulsada por el conocimiento se ha convertido en un factor decisivo para la competitividad de las naciones y de las empresas, son particularmente inquietantes los deficientes resultados logrados por las instituciones hospitalarias públicas tipo II de La Guajira colombiana en materia de innovación. En este trabajo se han abordado los aspectos relacionados con los tipos de innovación, innovación de procesos e innovación de naturaleza funcional, aplicando un marco metodológico que permitió conocer las prácticas de las instituciones objeto de la investigación en innovación y modernización tecnológica. 
Teniendo en cuenta los resultados obtenidos, con relación a la innovación incremental, se pudo concluir, según lo referido por los informantes clave, que en las instituciones públicas hospitalarias tipo II, el desarrollo de actividades de I $+\mathrm{D}$ se encuentra en un punto medio, acercándose hacia lo positivo; podría afirmarse que están dando los primeros pasos para involucrarse más en procesos de innovación que les permita garantizar que la tecnología existente está acorde con los requerimientos de la institución y satisfaga la necesidad de sus clientes.

$\mathrm{Al}$ respecto, Machado (1999) señala que la innovación incremental se trata de pequeños cambios dirigidos a incrementar la funcionalidad y las prestaciones de la empresa, que, si bien aisladamente son poco significativas, cuando se suceden continuamente de forma acumulativa pueden construir una base permanente de progreso. Así, se observa cómo el crecimiento y el éxito experimentado por las empresas de automoción en los últimos tiempos responde, en gran parte, a programas a largo plazo, caracterizados por una sistemática y continua mejora en el diseño de productos y procesos.

En relación con la innovación radical, se pudo establecer, que en las instituciones hospitalarias públicas tipo II de La Guajira colombiana, ésta no representa relevancia para mejorar o ajustar lo ya existente, de- bido a que deben regirse por normas establecidas para el sector salud por el Estado colombiano. Esto es aceptable si se tiene en cuenta que Machado (1999) afirma que la innovación radical implica una ruptura con lo ya establecido. Son innovaciones que crean nuevos productos o procesos que no pueden entenderse como una evolución natural de los ya existentes.

En relación con el indicador innovación original, según lo referido por los informantes clave, se pudo deducir que este aspecto no ha influido para el mejoramiento de la eficacia del personal. Podría pensarse que se debe a la apatía de los funcionarios, debido a que la mayoría manifestaron estar en desacuerdo o a una inadecuada implementación de la misma, como se pudo observar pues más del $70 \%$ de los informantes clave afirmó estar totalmente en desacuerdo. Lo anteriormente expuesto propicia que no se aproveche la capacidad de creatividad y talento del recurso humano contratado al interior de la institución. Con respecto a este indicador, Downs y Mohr (1976) afirman que las empresas deben concebir la innovación original como toda idea práctica u objeto que se percibe como novedoso a pesar de que incluso pueda ser una rutina para otras empresas.

En cuanto al indicador innovación adaptada, se pudo deducir, por el total de informan- 
tes claves que opinaron estar medianamente de acuerdo con este indicador, que en las instituciones públicas hospitalarias, a pesar de éstas regirse por normas específicas del sector salud, al momento de aplicar las innovaciones impuestas por el órgano rector, éstas se realizan de manera informal, es decir, no se acata en un 100\% lo establecido al aplicar las innovaciones solicitadas, ya sea en materia de mejoramiento de procesos o transferencia de tecnología, lo que ha ocasionado, en la mayoría de las instituciones, que este proceso fracase o se quede a medias.

Sobre este indicador, Baumol (1993) opina que también son innovaciones las actividades de transferencia de tecnología que aprovechan las oportunidades de introducir una tecnología ya disponible y válida (normalmente con alguna modificación que la adapte a las condiciones locales) en áreas geográficas cuya aptitud para la misma no había sido anteriormente reconocida ni utilizada.

\section{Dimensión: innovación de procesos.}

La dimensión innovación de procesos se analizó a través de los siguientes indicadores: innovación en cadena, eslabón, internalizar externalizar y roles personales. Para el indicador innovación en cadena, se pudo deducir que los informantes clave en las instituciones hospitalarias sí determinan los cambios en los procesos ya que un alto porcentaje respondió estar de total y mediana- mente de acuerdo, esto les permite convertir problemas y retos en ventajas competitivas, con el objetivo de diferenciarse frente a la competencia; los insumos más importantes se encuentran en indicadores y encuestas de calidad, con los que se miden los índices de satisfacción al cliente y se identifican los problemas y retos del grupo, para así enfocar los planes de acción para construir ventajas competitivas, como lo expresa Fernández (2005).

Con respecto al indicador proceso eslabón, en el ítem sobre si se realizan chequeos a las herramientas de gestión de procedimientos administrativos y medioambientales, se puede considerar que los informantes clave, mostraron diversidad de criterios al momento de escoger las opciones de respuestas, pero la tendencia muestra que las instituciones revisan su gestión y los procedimientos implementados.

Según Fernández (2005), son vías que conectan las tres áreas relevantes del proceso de innovación tecnológica, la investigación, el conocimiento y el proceso de cadena central de innovación tecnológica. Lo anterior, está ligado a la capacidad de crear empresas innovadoras con alto potencial de crecimiento económico y de generación de empleos de calidad. Es así que muchos países han estado trabajando en la construcción de un sistema de emprendimiento para fomentar 
un esquema masivo de creación de nuevas empresas.

Frente al indicador internalizar procesos, la mayoría de los informantes clave respondieron estar mediana y totalmente en desacuerdo, debido a que las instituciones hospitalarias objeto de la investigación sí utilizan servicios temporales para desarrollar procesos, es decir, entregan a terceros el manejo de procesos misionales, ocasionando esto que se deteriore la calidad de algunos servicios por la falta de pertenencia con la institución si tenemos en cuenta que, para Fernández (2005), internalizar procesos consiste en fichar a personal clave, incluso a equipos enteros, para realizar dentro de la empresa funciones cruciales que los proveedores externos no logran satisfacer. Además, la internalización es una estrategia de negocio que consiste en integrar y consolidar dentro de la empresa determinadas áreas críticas.

Por otra parte, al analizar los resultados relacionados con el indicador externalizar procesos y referidos por los informantes clave, se hace necesario que las instituciones realicen tareas de apoyo con empresas prestadoras de servicios. Lo anteriormente señalado propicia que se alcancen los objetivos necesarios para responder a las necesidades sugeridas para el desarrollo de los procesos. Según Quélin (2000), externali- zar los procesos es un movimiento que depende de una decisión de la dirección general que afecta al fundamento del oficio de la empresa y a su perímetro de actividad. Por lo tanto, al lado de las numerosas ventajas, también son numerosos los riesgos, en particular en términos de dominio de las competencias y del saber hacer, de dependencia a largo plazo y de mantenimiento del contrato que une la empresa a su prestador de servicio.

En el estudio de la dimensión roles personales hay igualdad de criterios entre los informantes clave al momento de escoger las opciones de respuestas, mostrando una equivalencia. Se puede afirmar que en las instituciones hospitalarias todos los funcionarios no se benefician de los programas de capacitación, no les ofrecen oportunidades para desarrollar sus habilidades y el personal para desarrollar investigaciones de I + D no es lo suficientemente calificado. Lo anterior, puede generar ineficacia en los procesos e ineficiencia en los recursos. Estos roles, considera Fernández (2005), que deben ser desempeñados por los individuos que desempeñan puestos clave para el reconocimiento de potencial de una innovación, es decir, el papel que desarrolla cada uno.

Dimensión: innovación en naturaleza funcional. En el estudio de la dimensión innovación en naturaleza funcional, 
se inicia el análisis de identificar la naturaleza funcional a través de los siguientes indicadores: tecnológicas, comerciales, administrativas, financiera y recursos humanos. Al considerar los resultados presentados, con relación a la naturaleza funcional, en el indicador tecnológica y según los informantes clave, se presenta una situación positiva en cuanto al grado de novedades de los servicios. Lo anterior puede tomarse como una fortaleza para las instituciones hospitalarias, ya que cuentan con tecnologías apropiadas, así como también con tecnología de punta para prestar un mejor servicio a sus clientes.

Se puede considerar sobre el indicador comercial, que son inexistentes las estrategias para la venta de los servicios, ya que un alto porcentaje de los informantes clave respondieron desfavorablemente. Esto podría deberse a la falta de políticas en las instituciones que impacten las competencias dentro del mercado, trayendo como consecuencia una debilidad en la institución, sobre todo si se considera que Drucker (2000) afirmó que las innovaciones comerciales deben también manifestadas a través de nueva presentación de un producto o de una nueva forma de distribución de un producto, es decir, una nueva campaña publicitaria.
Para el indicador administrativo, el estudio dejó apreciar que los objetivos son aplicados a las personas que laboran en las instituciones hospitalarias, ya que se aplican normas y mecanismos y se intercambian procedimientos entre sí, logrando correspondencia con Fernández (2005), quien opina que las innovaciones administrativas deben coincidir con los cambios radicales que se refieren al sistema administrativo de una organización y la relación entre las personas que interactúan para cumplir con los objetivos de la misma; cualquier innovación implica a su vez que estén intrínsecamente asociadas a las innovaciones técnicas.

Referente al indicador financiera, la tabulación refleja la existencia de barreras para los aspectos relacionados con la venta de servicio que prestan estas instituciones hospitalarias. Esto demuestra la ausencia de procesos que se generan en las mismas. Con relación a este indicador, Drucker (2000) se refiere a que éste hace referencia a aquellos aspectos relacionados con la compra/venta, que, por su carácter diferente e inédito, conceden a la empresa una originalidad sobre el resto de las empresas que compiten en el mercado; a veces, a la introducción de un nuevo producto o servicio en el mercado, el cual conlleva a cambiar las prácticas comerciales actuales. 
Con respecto al indicador recursos humanos, el personal en las instituciones hospitalarias no tiene las mismas oportunidades de introducir mejoras o cambios, ya que la mayoría de las respuestas de los informantes clave son desfavorables, poniendo en evidencia que hay una falta de formación y capacitación en estas instituciones para el aprovechamiento de oportunidades y, por ende, debilidad en el mejoramiento de competencias del recurso humano. Fernández (2005) sugiere la introducción de nuevas políticas de recursos humanos, tanto en lo relativo a la selección, con objeto de atraer mejores trabajadores, como en lo relacionado con la motivación y la retención del personal para así mejorar su productividad y apropiarse parte de los beneficios resultantes.

Las empresas constituyen el elemento fundamental en el que interactúan personas con diferentes tipos de conocimientos profesionales y técnicos, combinando su acción con el objeto de lograr resultados colectivos.

\section{Conclusiones y recomendaciones}

Las empresas constituyen el elemento fundamental en el que interactúan personas con diferentes tipos de conocimientos profesionales y técnicos, combinando su acción con el objeto de lograr resultados colectivos. En función de ello, el analizar los aspectos relacionados con los tipos de innovación: in- novación de procesos e innovación de naturaleza funcional permitió determinar las prácticas en innovación y modernización tecnológica en las instituciones hospitalarias de La Guajira-Colombia, en consecuencia, la capacidad de las empresas para innovar se relaciona estrechamente con la forma en que se constituye, se genera y se utiliza el conocimiento, de manera que cualquier análisis debe incorporar categorías conceptuales que examinen la forma en la que las empresas llevan a cabo el proceso de innovación.

Por lo antes expuesto, y teniendo en cuenta los resultados y lo concluido, se plantean las siguientes recomendaciones:

- Difundir los lineamientos teóricos a las autoridades competentes de los hospitales Nuestra Señora de los Remedios en Riohacha y Hospital San José de Maicao en La Guajira colombiana.

- Ejecutar los lineamientos teóricos, en los hospitales Nuestra Señora de los Remedios en Riohacha y Hospital San José de Maicao en La Guajira colombiana.

- Fortalecer la innovación en los hospitales Nuestra Señora de los Remedios en Riohacha y Hospital San José de Maicao en La Guajira colombiana. 
- Desarrollar futuras investigaciones sobre innovación en empleados medios de los hospitales Nuestra Señora de los Remedios en Riohacha y Hospital San José de Maicao en La Guajira colombiana.

- Aprovechar el recurso humano calificado contratado en las instituciones hospitalarias públicas tipo II en el desarrollo de actividades de investigación y desarrollo (I y D).

- Difundir las políticas destinadas a promover la modernización tecnológica, definidas para las instituciones hospitalarias públicas tipo II de La Guajira colombiana.

\section{Referencias}

Afuah, A. (1998). Innovation management. Strategies, Implementation, and Profits. Nueva York: Oxford University Press.

Ballestrini, M. (2001). Como se elabora el proyecto de investigación. México: Manual Moderno.

Basalla, G. (1988). The Evolution of technology. Cambridge: Cambridge University Press.

Baumol, W. (1993). Entrepreneurship, management, and the structure of payoffs. Cambridge: MIT Press.
Booz, E. Allen, J. y Hamilton, C. (1982). New Products management for the 1980's. Nueva York: Booz Allen Hamilton Inc.

Colciencias. (2005). Innovación: Aplicación de los conocimientos al desarrollo. Londres: Earthscan.

Downs, G. y Mohr, L. (1976). Conceptual issues in the study of innovations. $A d$ ministrative Science Quarterly, 21(4): 700-714.

Drucker, P. (2000). La administración en una época de grandes cambios. Buenos Aires: Sudamericana.

Fernández, E. (2005). Estrategia de Innovación. Madrid: Thomson.

Gaynor, G. (1999). Manual de gestión tecnológica. Bogotá: McGraw-Hill.

Hernández, R., Fernández, C. y Baptista, P. (2006). Metodología de la investigación. Cuarta edición. México: McGraw-Hill.

Machado, F. (1999). Administración eficiente de la innovación tecnológica en los países en desarrollo. Comercio exterior, 48(8). 607-616.

Mansfield, E. (1988). The speed and cost of industrial innovation in Japan and the United States: External vs Internal technology. Management Science, 34(10): 1157-1168. 
Martínez, C. (2000). Gestión e innovación tecnológica. Ingenieria y competitividad, 2(1). 65-71.

Méndez, C. (2001). Metodología, diseño y desarrollo del proceso de investigación con énfasis en ciencias empresariales. 4 ta ed. Bogotá: Limusa.

Pavón, J. y Goodman, R. (1981). Proyecto MODELTEC. La planificación del desarrollo tecnológico. Madrid: CDTI-CSIC.

Quélin, B. (2000). La “externalización” estratégica: ventajas y riesgos para la empresa. Recuperado de https://studies2.hec.fr/ jahia/webdav/site/hec/shared/sites/quelin/acces_anonyme/outsourcing/2000ejecutivosQuelin.pdf
Roberts, E. y Fusfeld, A. (1981). Staffing the innovative technology-based organization. Sloan Management Review, 22(3): 19-34.

Robertson, T. (1993). How to reduce market penetration cycle times. Sloan Management Review, 35(1): 87-96.

Tushman, M. y Anderson, P. (1986). Technological discontinuities and organizational environments. Administrative Science Qarterly, 31(3): 439-465. 